\title{
Prevención y tratamiento de la toxicidad digestiva
}

\author{
M. A. Pérez Escutia ${ }^{1}$, P. Samper Ots ${ }^{2}$, M. A. Cabeza Rodríguez ${ }^{1}$
}

\section{Introducción}

El índice terapéutico puede ser conceptualmente definido como el porcentaje de control tumoral dividido por el porcentaje de complicaciones. Controlando la tolerancia de los tejidos normales a la radiación podemos, no sólo incrementar la eficacia de la terapia antitumoral, sino también mejorar la calidad de vida del paciente disminuyendo los efectos secundarios.

La clave para reducir el riesgo de complicaciones, es la prevención. Para ello: 'Debemos conocer los factores relacionados con el paciente que pueden incrementar la toxicidad esperada del tratamiento radioterápico, y por tanto condicionar la planificación del mismo (disminución del volumen, reducción de dosis, e incluso abstención del tratamiento radioterápico, en el caso de indicaciones postoperatorias..). ${ }^{2}$ Se debe aplicar una técnica radioterápica depurada utilizando todos los medios técnicos a nuestro alcance para disminuir la toxicidad esperada del tratamiento. ${ }^{3}$ Finalmente valoraremos la utilización de métodos farmacológicos como antagonistas de la toxicidad, que modulan directamente la respuesta del tejido normal al daño citotóxico (modificadores de toxicidad, como la orgoteína, o los factores de crecimiento, o radioprotectores selectivos como la amifostina,... ).

Debemos diferenciar estas estrategias biológicas que ayudan a modular la respuesta, de los fármacos utilizados para el tratamiento de los síntomas producidos por la toxicidad radioinducida, fundamentalmente aguda (tratamiento de soporte), como son los antieméticos, analgésicos, antidiarreicos o protectores de mucosa, que nos ayudan a controlar la sintomatología, y a realizar el tratamiento radioterápico en condiciones óptimas.

Una vez establecida la toxicidad crónica, el tratamiento debe ser lo más conservador posible, y debe estar dirigido a

1 Servicio de Oncología Radioterápica. Hospital Universitario Doce de Octubre

2 Servicio Oncología Radioterápica. Hospital Central de Defensa

Madrid aliviar la sintomatología, y mejorar la calidad de vida de los pacientes.

En esta revisión nos vamos a referir exclusivamente a la toxicidad intestinal, derivada de la irradiación abdominal y pélvica, por ser la que verdaderamente condiciona el tratamiento radioterápico, ya que el intestino delgado es el principal órgano crítico y el limitante de dosis. Revisaremos en primer lugar las actuaciones relacionadas con la prevención de la toxicidad, y en un segundo lugar el tratamiento de las toxicidades aguda y crónica.

\section{Prevención toxicidad intestinal}

\subsection{Factores predisponentes de mayor toxicidad}

Edad: La edad por sí misma no constituye un factor predisponente de toxicidad ${ }^{1,2}$. Pero cuando se asocia a otros factores como cirugía previa, en el caso de los tumores de recto, sí que adquiere importancia, reflejando el compromiso vascular mayor que presenta este grupo de pacientes y que se traduce en una menor capacidad de recuperación de los tejidos sanos tras agresiones severas como son la cirugía y la radioterapia, con una mayor incidencia de toxicidad gastrointestinal severa ${ }^{3}$.

Diabetes mellitus, hipertension arterial: Estas patologías afectan de forma generalizada a la microvasculatura, comprometiendo la oxigenación tisular y por lo tanto inhibiendo la reparación de los tejidos irradiados. La diabetes así mismo también afecta a la viscosidad de la sangre promoviendo la agregación plaquetaria, y a la curva de disociación de oxígeno-hemoglobina, disminuyendo la tasa de liberación de oxígeno, todo esto contribuye a una mayor isquemia tisular y mayor incidencia de complicaciones tardías 2 .

Enfermedad inflamatoria intestinal: Los pacientes portadores de una enfermedad inflamatoria intestinal parecen presentar una peor tolerancia al tratamiento radioterápico con o sin quimioterapia. Su asociación con el cáncer colorrectal está bien documentada, y en el caso de tumores de recto de alto riesgo, existe indicación de administrar tratamiento oncológico quimio-radioterápico complementario, aunque hay que ser muy cuidadosos y emplear técnicas de minimización de irradiación de tejidos sanos (intestino delgado y grueso) $)^{4,5}$.

Estado general: El descenso en el performance status, 


\section{A. Pérez Escutia y cols.}

también conlleva una peor tolerancia al tratamiento, con un incremento de la complicaciones severas ${ }^{6}$. Por otra parte la desnutrición produce alteraciones en la estructura y función gastrointestinal, como pérdida de altura o ausencia completa de vellosidades intestinales, reducción de disacaridasas y aminopeptidasas en la mucosa digestiva y disminución de volumen y contenido enzimático de las secreciones gástricas, pancreáticas y biliares ${ }^{7}$.

Funcion intestinal previa al tratamiento: Se debe valorar muy cuidadosamente la función intestinal basal que presenta el paciente: movimientos intestinales al día, aerofagia, función rectal, ya que puede estar afectada por diversas causas (generalmente cirugías previas), y representa un compromiso intestinal ya establecido.

Cirugía previa: Pacientes que han tenido una laparatomía previa tienen mayor riesgo de tener toxicidad gastrointestinal, por el desarrollo de adherencias intestinales que presentan, que pueden prolapsarse en la pelvis, quedando expuestas a dosis plenas de radiación. Las adherencias debidas a cirugías previas tienen el mismo efecto ${ }^{6,8,9}$.

Quimioterapia: La quimioterapia previa o concurrente a la cirugía contribuye a aumentar la toxicidad aguda y crónica.

\subsection{Factores técnicos y planificación del tratamiento radioterapico}

\section{Intestino delgado}

El intestino delgado es el principal órgano de riesgo y el verdaderamente limitante de dosis, en la irradiación abdominal y sobretodo pélvica.

El volumen de los campos de tratamiento, y por tanto la cantidad de intestino delgado incluido en el campo de irradiación, la duración total del tratamiento, la dosis sesión, la energía de la irradiación, la técnica utilizada, y finalmente la dosis total administrada, son factores determinantes de la toxicidad del tratamiento radioterápico administrado. Sobre ellos debemos intervenir activamente, para conseguir el mejor índice terapéutico en cada caso.

Actualmente está plenamente establecido en la irradiación abdominal y pélvica, que volúmenes grandes, fraccionamientos no convencionales, fotones de baja energía, pocas puertas de entrada en la irradiación, incrementan de forma significativa la toxicidad severa digestiva y no son aceptables en los tratamientos radicales. Así por ejemplo, en el cáncer de recto, en el ensayo randomizado sueco 1 de radioterapia preoperatoria ${ }^{10}$, se utiliza una dosis/sesión de 5 Gy/día, durante 5 días, grandes campos de irradiación (límite superior del campo pélvico en la vértebra L2), y tan sólo dos puertas de entrada. Con esta técnica presentan una incidencia de obstrucción intestinal del 13.3\%. Sin embargo, cuando se utilizan 4 campos más reducidos, la toxicidad intestinal no se encuentra incrementada ${ }^{11}$. Por otra parte la importancia del fraccionamiento queda establecida cuando se analiza la toxicidad tardía del trato gastrointestinal, de los ensayos $\mathrm{H} 2$ y $\mathrm{H} 5$ de la EORTC de la enfermedad de Hodg$\mathrm{kin}^{8}$. En ellos se objetiva que los pacientes que se tratan con un fraccionamiento clásico (5x200 cGy), y no tienen laparatomía previa, tienen una baja incidencia de complicaciones (1\%), mientras que los pacientes que recibieron 3 fracciones semanales de 3.3 Gy después de laparatomía, presentaban una tasa de complicaciones del $39 \%$.
Además, hay que tener en cuenta, que el volumen de intestino delgado incluido en el campo de irradiación, es directamente proporcional a la incidencia de complicaciones tardías intestinales ${ }^{12}$, siendo esencial la administración de contraste en el intestino delgado durante la simulación de toda irradiación pélvica con carácter radical, para su máxima protección en todos los campos. Algunos autores llegan incluso a modificar el campo de tratamiento (descendiendo el límite superior del campo), cuando utilizan el contraste oral durante la simulación, obteniendo una disminución significativa de las complicaciones ${ }^{13}$.

También se dispone de una serie de técnicas de irradiación muy sencillas para disminuir la toxicidad intestinal durante la irradiación: por ejemplo el uso de múltiples campos (tres o cuatro), permite una protección de mayor cantidad de intestino delgado, comparado con la técnica de dos campos (AP-PA). El tratamiento de todos los campos diariamente, consigue una dosis biológicamente eficaz menor, para aquellas estructuras que sólo se incluyen en alguno de los campos. La utilización de campos laterales para la sobreimpresión, disminuyen la cantidad de intestino delgado en el campo de tratamiento. Y por último, la utilización de aceleradores lineales de alta energía $(>6 \mathrm{MV})^{14}$.

Actualmente con la incorporación de la radioterapia conformada tridimensional, se ha optimizado enormemente el tratamiento, al poder localizar con gran precisión en cada uno de los niveles del volumen de tratamiento, no sólo el PTV, sino también los órganos de riesgo, y disponer, en la evaluación final del tratamiento, de los histogramas dosis/ volumen.

Así mismo se puede llevar a cabo una serie de maniobras físicas para aumentar la exclusión de intestino delgado de la pelvis. La combinación de la posición de decúbito prono durante el tratamiento, con compresión de la pared abdominal y distensión vesical, consigue una reducción significativa en el porcentaje de intestino delgado incluido en el campo de irradiación respecto a la posición en decúbito supino ${ }^{15}$. El tratamiento en decúbito prono sin compresión de la pared no consigue de manera eficaz este desplazamiento. La utilización de Trendelenburg también resulta muy útil, especialmente en pacientes obesos ${ }^{16}$. Los sistemas de inmovilización con dispositivos similares al sistema belly board (dispositivos que llevan un orificio en la parte superior del campo de irradiación para permitir al intestino delgado desplazarse fuera del campo de radioterapia), cuando se trata en decúbito prono, disminuyen de manera muy importante el volumen de intestino delgado irradiado, en torno al $66 \%$, comparado con los pacientes tratados en decúbito supino y sin este dispositivo ${ }^{17}$

Las técnicas quirúrgicas también pueden contribuir a disminuir la toxicidad intestinal. Así por ejemplo, la colocación de clips en las áreas de alto riesgo, ayudan a definir mejor el volumen tumoral. Cualquier maniobra quirúrgica que excluya el intestino delgado de la pelvis es superior a dejar el suelo pélvico no peritonealizado, especialmente tras resección abdómino-pélvica. Entre ellas destacamos la colocación de mallas absorbibles de Dexón o vicrilo, que evitan temporalmente el descenso de las asas intestinales en pelvis ${ }^{18}$, o la reconstrucción del suelo pélvico.

Por último la dosis administrada constituye un factor fundamental en el desarrollo de toxicidad. Debemos, en todo momento, ser muy rigurosos con las dosis de tolerancia reco- 
mendadas, y cuando existan factores predisponentes de mayor toxicidad, tenerlo en cuenta para disminución de la misma.

\section{Intestino grueso}

Nos referimos fundalmentalmente a la proctitis como consecuencia de la irradiación de tumores ginecológicos, urológicos o de recto. Su desarrollo está relacionado directamente con la dosis rectal, y es volumen dependiente. Por tanto tiene una gran importancia en su prevención, todas las medidas que se puedan llevar a cabo durante la simulación y reproducción del tratamiento para minimizar el volumen rectal incluido: tener el intestino grueso vacío, algunos autores recomiendan el decúbito prono como posición de tratamiento, al considerar que de esta manera se reduce el volumen de recto que recibe altas dosis de radioterapia ${ }^{19}$, por otra parte para disminuir la variabilidad de posición de la próstata y las vesículas seminales durante el tratamiento, algunos autores recomiendan realizar la simulación y el tratamiento con la vejiga vacía, y por último, siempre hay que realizar una correcta inmovilización del paciente con cunas individualizadas.

Por otra parte, cuando se administran dosis elevadas, como en el caso de los tumores de próstata, la técnica radioterápica utilizada tiene gran trascendencia en el desarrollo de proctitis: con técnicas convencionales y escalada de dosis la incidencia de proctitis moderada-severa a 2 años, se incrementa de $20 \%$ en pacientes que reciben < o igual a 75 Gy, a $60 \%$ para aquellos que reciben dosis más elevadas. Con radioterapia tridimensional conformada, la toxicidad crónica intestinal desciende de forma significativa cuando se compara con la convencional, así en un estudio randomizado para dosis de $64 \mathrm{~Gy}$, la incidencia de proctitis y sangrado para el grupo de radioterapia convencional en relación con el de radioterapia conformada fue de $56 \%$ en relación a $37 \%$ para toxicidad grado $1(p=0.004)$, y de $15 \%$ en relación a $5 \%$ para toxicidad grado $2(p=0.01)$, respectivamente ${ }^{20}$.

El riesgo se incrementa cuando la dosis excede 70 Gy, correlacionándose con el volumen de la pared anterior rectal expuesto a dosis elevadas de radiación. De esta manera Centros de Referencia presentan una tasa actuarial de toxicidad rectal grado 2 a 3 años de $6 \%$ cuando se administran dosis entre 64.8-70 Gy, elevándose a 17\% cuando la dosis es de $75.6 \mathrm{~Gy}$.

Se han ideado técnicas más sofisticadas de radioterapia conformada, para administrar una dosis de 81 Gy, restringiendo la dosis rectal para no sobrepasar el V30 < o igual a $75 \mathrm{~Gy}$, comprobándose que no son eficaces, al presentar una toxicidad rectal del $15 \%$.

La radioterapia de intensidad modulada, por el contrario, sí ha contribuido en los tumores prostáticos a una mejora en la conformación de los campos, y a una reducción del recto irradiado, lo que se traduce en un descenso significativo de la toxicidad rectal. De esta manera es posible una escalada de dosis de forma segura a $86.4 \mathrm{~Gy}$, con un descenso significativo de la toxicidad rectal ( $5 \%$ toxicidad grado 2 , no toxicidad grado 3 , con un seguimiento medio de 36 meses $^{21}$.

Cuando se utiliza braquiterapia de forma exclusiva o asociada a radioterapia externa, como sucede en los tumores prostáticos o ginecológicos (endometrio y cervix fundamentalmente), para conseguir el máximo control local con la mí- nima morbilidad, es necesario una correcta colocación de los aplicadores, en ocasiones la realización de diferentes maniobras para desplazar el recto y alejarlo del volumen de interés, como retractores rectales, gasas radiopacas, etc, y sobretodo seguir las recomendaciones internacionales en cuanto a prescripción de dosis ${ }^{22,23,24}$.

\subsection{Recomendaciones dietéticas}

Las alteraciones gastrointestinales que se producen durante la irradiación de la cavidad abdominal y pélvica, se producen precozmente, detectándose tras dosis bajas de irradiación. Los cambios histológicos consisten en disminución de las vellosidades intestinales, y producen alteraciones en la absorción de los alimentos, con mal absorción de grasas, lactosa y sales biliares en el colon que inhiben la absorción de agua. Además se produce una hipermotilidad intestinal.

Por ello es conveniente introducir una serie de recomendaciones dietéticas precozmente, antes que se establezcan los síntomas clínicos de la gastroenteritis, mejorando así, la tolerancia y disminuyendo de forma significativa la intensidad de los síntomas.

Se debe aumentar el número de ingestas diarias (se recomienda al menos 5), disminuyendo la cantidad en cada una de ellas. Así mismo se recomienda una dieta elemental, baja en residuos, baja en grasas y en lactosa ${ }^{25}$. Se debe reducir de forma profiláctica la ingesta de alimentos con alto contenido en fibra insoluble: alimentos integrales, verduras o frutos secos. La fruta se puede administrar sin piel, cocida, al horno, en almíbar, en zumos colados (no de cítricos), en forma de membrillo o rallada (manzana). Las pectinas y los taninos de algunas frutas pueden ejercer efectos beneficiosos en la regulación del tránsito intestinal.

También se debe disminuir la cantidad de grasa de la dieta, y se recomienda reducir la ingesta de leche para que haya un menor aporte de lactosa. Se recomienda la ingesta de derivados lácteos como el yogur que posee un contenido en lactosa, y un efecto beneficioso sobre la flora intestinal

Hay una serie de nutrientes que podemos aportar en la dieta, y que podrían contribuir a mejorar el daño intestinal radioinducido. Son los siguientes:

\subsubsection{Glutamina}

La glutamina es un aminoácido encargado de mantener la integridad de la pared intestinal. Durante las situaciones hipermetabólicas, se produce una severa depleción de glutamina debido a un elevado consumo mediado en parte por el cortisol, como hormona fundamental en situaciones de estrés, que no puede compensarse por una mayor producción, aumentando sus necesidades espectacularmente ${ }^{26}$.

En estas condiciones la glutamina puede convertirse en un nutriente esencial, y su deficiencia puede comprometer la función inmunitaria, el equilibrio ácido base y la integridad del enterocito, facilitando la producción de una translocación bacteriana.

Durante la irradiación abdominal y pélvica, estudios preclínicos demuestran que protege la integridad de la mucosa del tracto gastrointestinal alto y bajo, de los efectos de la radioterapia disminuyendo la intensidad de la enteritis ${ }^{27,}{ }^{28}$. Los estudios clínicos son escasos y de poco valor, por el limitado número de pacientes que incluyen y por la diversidad de dosis y esquemas utilizados 29,30 . En irradiación abdominal y 


\section{A. Pérez Escutia y cols.}

pélvica hay sólo dos estudios: el primero incluye 26 pacientes con tumores de próstata en los que se administra radioterapia, y son randomizados a recibir 21 gramos/día de glutamina (7 gramos 3 veces/día), o placebo de manera profiláctica. Aunque no hay diferencias en el número, aspecto y consistencia de las heces, las biopsias realizadas antes y 11 días después de la radioterapia, reflejan mejorías significativas en el grupo que recibió glutamina ${ }^{31}$. El segundo estudio se lleva a cabo por el Grupo de Tratamiento del Cáncer del Norte y Centro (NCCTG). Es un estudio fase III randomizado y doble ciego, en 129 pacientes de diferentes Instituciones que son sometidos a irradiación pélvica. Los pacientes recibieron glutamina 4 gramos/2 veces al día (8 gramos/día), o placebo, comenzando desde el inicio de la radioterapia y continuando dos semanas después de finalizar. No se objetivaron diferencias en toxicidad digestiva ni en calidad de vida, aunque fue bien tolerado ${ }^{32}$.

Es posible que en condiciones de estrés severo, como sucede en la irradiación pélvica, se necesiten dosis más elevadas de glutamina para mantener la integridad del intestino. Algunos autores indican dosis entre 20-40 gramos/día ${ }^{33}$. Una dosis prudente sería 30 gramos/día, que debe administrarse en 3 dosis, siendo recomendable se inicie la administración 7 días antes del inicio de la radioterapia, y se continúe hasta 2 semanas después de haber finalizado. En enfermedades gastrointestinales crónicas, como podría ser la enteritis por radiación podría usarse una dosis de mantenimiento de 10 gramos/día vía oral.

Sí parece que su administración en situaciones de irradiación abdominal y pélvica es bien tolerada.

Es recomendable la realización de estudios clínicos bien diseñados que ayuden a determinar su eficacia en la prevención de la enteritis.

\subsubsection{Arginina}

Se considera también un aminoácido semiesencial, que parece estimular la respuesta inmune y el balance nitrogenado, y junto con la glutamina soporta la barrera mucosa intestinal. Existen numerosos estudios clínicos, no controlados que demuestran que la adición de arginina a fórmulas estándar, mejoran la respuesta inmunitaria.

Estudios preclínicos en ratas que son sometidas a irradiación abdominal, demuestran que la administración de una dieta enriquecida con arginina, tiene efectos protectores sobre la mucosa intestinal, mejorando la translocación bacteriana, y manteniendo el número y la altura de las microvellosidades intestinales ${ }^{28}$.

No se disponen de estudios clínicos en irradiación abdominal, y por lo tanto se desconoce su eficacia en estas circunstancias. La dosis recomendada es de $500 \mathrm{mg} / \mathrm{kg} / \mathrm{día}$, lo que equivale en un adulto a unos 25-30 gramos/día. No está claro cómo se debe administrar en relación con la radioterapia (parece que la administración pre y post-radioterapia, no posee una protección superior a la administración posterior.

\subsection{3. Ácidos grasos}

Los lípidos poseen funciones bioquímicas estructurales y reguladoras muy importantes, más allá de su función energética.

Los ácidos grasos de cadena muy larga y muy insaturados, de la serie omega 3: eicosapentanoico (EPA), y docosa- hexanoico (DHA), actuan regulando la producción de citoquinas, que son: mediadoras de la inflamación, contribuyen a la patogenia de enfermedades degenerativas crónicas, y mediadores de la respuesta aguda al estrés. En voluntarios sanos se ha comprobado que tienen una acción antiinflamatoria, inmunoestimuladora, vasodilatadora y antiagregante, reduciendo la severidad del daño inflamatorio.

Por otro lado, el ácido gamma linolénico, un ácido graso esencial, poliinsaturado, y de la serie omega 3, pero no proveniente de los pescados, posee una acción vasodilatadora, antiagregante, inhibe la liberación de radicales libres y la quimiotaxis de neutrófilos ${ }^{26}$.

Por otra parte, los ácidos grasos de cadena corta poseen efectos beneficiosos sobre la mucosa intestinal y ejercen una acción inmunomoduladora más allá del mantenimiento de la mucosa intestinal.

\subsubsection{Fibra soluble}

La administración de una dieta con fibra soluble (alimento prebiótico), es beneficiosa, pues activa la fermentación intestinal, normalizando la flora intestinal y haciendo que las bacterias anaérobicas sean las predominantes, lo que se cree que tiene un papel protector de la mucosa intestinal. Aumenta la producción de ácidos grasos de cadena corta, que promueven la proliferación de las células epiteliales de la mucosa del intestino delgado y grueso, mejorando la atrofia, y la absorción de agua, con lo que mejora la consistencia de las heces y los movimientos intestinales ${ }^{34}$.

\subsubsection{Preparados bacterianos probióticos}

Se ha demostrado que la administración de alimentos bacterianos probióticos (lactobacilo o similar) poseen un efecto beneficioso sobre el trato gastrointestinal. Disminuyen la apoptosis, modulan la actividad inflamatoria y la producción de citoquinas, alteran la composición y el metabolismo, así como las propiedades funcionales de la flora entérica, y preservan la integridad de la barrera intestinal. Todo ello tiene un efecto protector de las alteraciones producidas por la radioterapia, que tienen relación con alteraciones tanto cuantitativas como cualitativas de la microflora intestinal bacteriana $^{35}$.

\subsubsection{Suplementos nutricionales}

En pacientes sometidos a irradiación abdominal, a veces es necesaria la administración de suplementos nutricionales para complementar una dieta oral insuficiente, o por problemas de absorción intestinal.

Salvo contraindicaciones, las dietas pueden ser polímeras estándar y de alta densidad calórica, para poder aportar en menos volumen más calorías y facilitar así el cumplimiento de la prescripción.

La presentación y el sabor debe individualizarse.

Suelen presentar una combinación de nutrientes y estar enriquecidas con EPA y DHA, por su efecto beneficioso sobre el estado nutricional del paciente ${ }^{36}$.

\subsection{Sucralfato}

El sucralfato es un complejo de hidróxido de aluminio, que no se absorbe sistémicamente, y que es eficaz en el tratamiento de las úlceras gastrointestinales.

In "vitro" forma un complejo con proteínas en la mucosa 
dañada dando lugar a una capa protectora. Protege las superficies mucosas al unirse con el tejido ulcerado formando una barrera, mediante la producción de prostanglandinas, así mismo al unirse a factores de crecimiento, y por último, a sus efectos angiogenéticos ${ }^{37}$. Se ha postulado que este efecto podría contribuir a reducir el daño microvascular, y por tanto mejorar la toxicidad crónica.

Las ventaja de su utilización es la simplicidad de su administración y la baja frecuencia de efectos secundarios.

Se ha utilizado en la prevención del daño mucoso y cutáneo radio-inducido en otras partes del tracto digestivo superior como consecuencia de la irradiación de tumores de cabeza y cuello o el área torácica. También para mejorar la mucositis que se produce tras la quimioterapia.

En el área abdominal y pélvica se dispone de 6 estudios que evalúan el beneficio del sucralfato vía oral en la prevención de la enteritis y proctitis debidas a la radioterapia.

Todos ellos son randomizados y a doble ciego, e incluyen pacientes sometidos a irradiación de toda la pelvis, evaluándose en éstos, la enteritis y la proctitis ${ }^{38-42}$, o bien de volúmenes más pequeños en el caso de tumores de próstata, donde tan sólo se evalúa la proctitis ${ }^{43}$. La dosis utilizada más habitualmente es de 6 gramos/día por vía oral, comenzando generalmente con el inicio de la radiación, aunque en algún estudio se inicia 1-2 semanas antes del tratamiento ${ }^{38,40}$.

Generalmente se evalúa la toxicidad aguda, aunque en dos de ellos ${ }^{37,45}$ se valora también la toxicidad a los 12 meses.

Los resultados son contradictorios, y tan sólo el estudio sueco de Henrikson ${ }^{38}$ y el español de Valls ${ }^{40}$ presentan mejorías significativas en el grado de enteritis aguda (valorado por el descenso en el número de deposiciones, porcentaje de heces diarréicas o uso de loperamida), cuando se compara con la rama que recibe placebo. En el resto de los estudios no se aprecian diferencias significativas. La proctitis ni siquiera en los anteriores estudios mejora, y en el estudio de Materson ${ }^{41}$, que utiliza la misma dosis (6 gramos/día), empeora con la administración de sucralfato, objetivándose un incremento de la incontinencia rectal y de la necesidad de protectores. Este mismo estudio también objetiva una mayor incidencia de intolerancia gastrointestinal con la administración del fármaco.

Una posible explicación puede ser que el producto no alcance el tracto digestivo inferior, o que llegue muy diluido como para ejercer ese efecto protector. Si esto fuera así, cuando se administra por vía rectal, en enemas, sí que se objetivarían mejorías significativas. En el estudio australiano de O'Brien ${ }^{44}$, se utiliza vía endorectal y tampoco se objetivan diferencias significativas en el grado de proctitis agudas. Una razón para justificar esta falta de eficacia podría ser que necesitara un medio ácido, para llegar a ser activo, que no presenta el recto.

También puede haber problemas de unión a la mucosa dañada. En el estudio de Taal, se utiliza sucralfato marcado con Tecnecio-99, y se administra a 26 pacientes que presentan esofagitis radioinducida. Sólo el $31 \%$ de los pacientes tiene la droga activamente unida al tejido dañado, y la actividad radioactiva sólo se objetiva en el $15 \%$ de los pacientes a las 1 y 2 horas $^{45}$.

Respecto a la toxicidad crónica, evaluada en tres estu$\operatorname{dios}^{38,46,47}$, parece mejorar el grado de proctitis, aunque sólo alcanza significación estadística en el estudio sueco de
Henrikson ${ }^{38}$. Esto avalaría su acción angiogenética y de mejora de la microvasculatura tisular.

Podemos concluir con que, si bien los estudios de que se disponen en la actualidad no presentan resultados concluyentes, quizás por el escaso número de pacientes que incluyen, las ventajas teóricas que presenta el sucralfato, le confiere un gran interés como citoprotector, siendo necesario la realización de más ensayos clínicos que más claramente sus ventajas.

\subsection{Orgoteina}

La orgoteina es el nombre genérico adoptado en 1971 para la versión medicamentosa de la superóxido dismutasa de Cu-Zn. Actúa a nivel extracelular, inhibiendo la fase aguda inflamatoria mediada por los radicales libres, produciendo la dismutación de los radicales de superóxido, para convertirlos en oxígeno molecular y agua, no tóxicos, restaurando la competencia tisular, al detener el ciclo tóxico.

Diferentes estudios han demostrado su eficacia en el tratamiento de los efectos agudos radioinducidos 49,50 .

Hay experiencias previas en otras localizaciones de reducción de la fibrosis establecida ${ }^{50}$.

El papel de la orgoteína en la prevención de los efectos fibróticos tardíos, se basa en la hipótesis de que los eventos en la inflamación aguda pueden desarrollar fibrosis, y de que usando la orgoteína en el tiempo de máxima reacción inflamatoria, actuaría sobre uno de los mecanismos involucrados en la fibrosis radioinducida, y podría mejorarla.

Basado en esta hipótesis, Esco lleva a cabo un estudio randomizado, para demostrar su eficacia en la prevención de la toxicidad tardía gastrointestinal y urinaria tras radioterapia pélvica ${ }^{51}$

Se incluyen 100 pacientes con cáncer de recto operados, y sometidos a tratamiento quimio-radioterápico postoperatorio. Al finalizar la radioterapia, son randomizados a observación, o a recibir orgoteína $8 \mathrm{mg} /$ día i.m., 3 días/semana durante 7 semanas. Los pacientes son seguidos durante 2 años.

Los resultados muestran que la administración de orgoteína una vez finalizado el tratamiento radioterápico, previene de forma segura y significativa la toxicidad tardía a nivel pélvico. Esta disminución es particularmente manifiesta a nivel intestinal, de tal manera que los pacientes no tratados con orgoteína tienen de forma significativa 10.5 veces más posibilidades de desarrollar toxicidad intestinal, que los tratados, con una reducción del riesgo relativo de toxicidad tardía relevante (grado 2 o superior), a los 12 meses de 7.16 veces, a los 18 de 11.4 veces, y a los 24 meses de 10.5 meses. También se demuestra una asociación significativa $(p<$ 0.0001 ), entre pacientes con toxicidad aguda relevante, y la toxicidad tardía relevante, existiendo una coincidencia en la localización. Por otra parte la orgoteina no ha mostrado ningún tipo de efecto radioprotector sobre el control tumoral, y por último su tolerancia es excelente. Este estudio abre una nueva vía en la utilización de la orgoteína, y en la realización de estudios controlados que confirmen estos resultados.

\subsection{Amifostina}

Es un citoprotector selectivo de los tejidos normales. Elimina los radicales libres oxigenados que resultan de la irradia- 


\section{A. Pérez Escutia y cols.}

ción, por lo que previene la formación de los peligrosos radicales hidrperóxidos que dañan el DNA e incrementan el riesgo de muerte celular.

Estudios randomizados han demostrado que esta droga reduce la severidad y la duración de la xerostomía en pacientes con tumores de cabeza y cuello ${ }^{52}$, la esofagitis y neumonitis radioinducida en el cáncer de pulmón ${ }^{53}$, sin comprometer la efectividad en el control tumoral. Por el contrario la mayoría de los datos clínicos sugieren que los pacientes que reciben amifostina con quimio - radioterapia tienen mejor tasas de control. Este beneficio se puede atribuir a varias razones: 1. protección del régimen terapéutico por sí mismo, por lo que se puede llevar a cabo el tratamiento oncológico previsto sin demoras. 2. Eventual interferencia de la amifostina con el sistema inmune protegiendo la inmunidad celular. 3. Modulación de la respuesta biológica, evitando la formación de metástasis.

La eficacia de la administración intravenosa en pacientes sometidos a irradiación pélvica, se ha valorado en diversos estudios randomizados, aunque de escaso número de pacientes. Los resultados demuestran una reducción significativa de la mucositis rectal, epitelitis perineal aguda, y toxicidad vesical, comparado con el grupo control ${ }^{54,55}$

Recientemente se han publicado los resultados del estudio del Grupo Helénico de Oncología Radioterápica ${ }^{56}$, que incluye un total de 205 pacientes, con tumores pélvicos (32 con tumores de recto, 47 de vejiga, 40 de próstata, y 86 ginecológicos), randomizados a recibir radioterapia sola, o radioterapia con amifostina. La amifostina se administró a dosis de $340 \mathrm{mg} / \mathrm{m}^{2}$ i.v., 15 minutos antes de la radioterapia, con antieméticos estándar 30 minutos antes. Todos los pacientes recibieron radioterapia convencional, a dosis radicales (65-70 Gy), o postoperatoria (50 Gy), con 45 Gy a toda la pelvis con un fraccionamiento de 5x180-200 cGy. Se evaluó la toxicidad cutánea, intestinal, vesical, y hematológica de acuerdo a la escala de la RTOG/EORTC. Los resultados muestran una reducción significativa de la toxicidad gastrointestinal inferior: el $22.1 \%$ de los pacientes del grupo control presentaron toxicidad Grado 2-3 en la tercera semana del tratamiento, comparado con el $5.5 \%$ del grupo de la amifostina. A las 6 semanas después de finalizar el tratamiento se mantenía la reducción: 3 pacientes en el grupo de la amifostina (2.9\%) presentaban toxicidad grado $2-3$, comparado con 6 pacientes $(6.7 \%)$ del grupo control $(p=0.308)$. Durante la media de seguimiento de $12 \mathrm{me}$ ses, se observó poca toxicidad grado 2-3 en ambos grupos ( 1 en el grupo control, y 4 en el de la amifostina, uno de los cuales necesitó cirugía por obstrucción intestinal). También se comprobó una reducción significativa en la toxicidad urinaria entre ambos grupos, documentada al final de la cuarta semana de tratamiento: 13.7 pacientes de grupo control presentaron toxicidad grado $2-3$, comparado con $4.5 \%$ del grupo de la amifostina $(p=0.043)$. La amifostina fue muy bien tolerada, con tan sólo 1 paciente que requirió suspensión de la misma por hipotensión. No hubo, por otra parte diferencias significativas en la respuesta tumoral, valorada a las 6 semanas de finalizar el tratamiento: la respuesta clínica (respuestas completas y parciales) fue de $96.8 \%$ en el grupo de la radioterapia sola, frente a $98.3 \%$ en el de la amifostina.

Las consideraciones económicas son un importante factor en el uso de la amifostina. Los citoprotectores reducen los efectos secundarios debidos al tratamiento, y por ello aumentan la calidad de vida de los pacientes, más que la supervivencia global o la cantidad de vida. En este estudio no se evalúa la calidad de vida de los pacientes como un instrumento válido y real del coste-efectividad de la amifostina, siendo recomendable, en el futuro se realicen estudios que incorporen estos aspectos. Los autores concluyen con que se requieren estudios prospectivos randomizados, estratificados por localización y estadificación tumoral para confirmar estos resultados.

La administración subcutánea de amifostina se evalúa en el estudio del grupo griego de Koukourakis ${ }^{57}$, que incluye 140 pacientes ( 40 con tumores pélvicos, 60 con tumores torácicos, y 40 con tumores de cabeza y cuello), que fueron randomizados a recibir radioterapia radical con o sin amifostina subcutánea (dosis de $500 \mathrm{ng}$, diluidos en $2.5 \mathrm{ml}$ de suero salino, inyectados subcutáneamente, 20 minutos antes de cada sesión de radioterapia). Los pacientes con tumores ginecológicos recibieron radioterapia externa (50 Gy), seguida de sobreimpresión con braquiterapia (70 Gy al punto A). Entre los pacientes que recibieron amifostina, el $85 \%$ la toleró bien, el $16 \%$ presentó astenia severa, y en 6 se objetivó fiebre alta y rash cutáneo. Globalmente hubo que suspender el tratamiento en el $14 \%$ (un 5\% por astenia severa, y en $10 \%$ debido a fiebre o rash cutáneo). En esta vía de administración no se objetivó hipotensión. En cuanto a la mejoría en la tolerancia a la radioterapia, la administración de amifostina, redujo la incidencia de mucositis esofágica o rectal, grado 2 (13\% en relación con 35\%) y grado $3-410 \%$ en relación a $15 \% \mathrm{p}<0,05)$, no habiendo suspensiones o retrasos en la administración de la radioterapia en todas las localizaciones. También se redujo la toxicidad cutánea perineal/ vulvar, presentando toxicidad grado 2-3 todos los pacientes que no recibieron la amifostina, en relación a sólo 1 paciente del grupo de la amifostina con toxicidad grado 1. Por otra parte, también hubo una disminución en la incidencia de cistitis (en 0 pacientes en relación a 7 pacientes grado 2) y xerostomía (58\% en relación a $75 \%$ ). La respuesta al tratamiento sólo se evaluó en pacientes con cáncer de pulmón y de cabeza y cuello, y no hubo diferencias significativas. El estudio concluye con que la administración subcutánea de amifostina es bien tolerada, efectiva, reduce la toxicidad aguda y previene la crónica. Su administración es más simple, y segura, en relación con la intravenosa, ahorrando tiempo. Recomienda continuar con estudios, estratificados por localización tumoral.

Por último, la vía endorectal, es una vía de notable interés, como forma de incrementar la diferencia en concentración del radioprotector del tejido sano en relación al tumoral. Estudios preclínicos muestran un acúmulo preferencial de amifostina en la mucosa rectal, sin evidencia de la misma en la circulación sanguínea. A pesar de todo esto, hay poca experiencia. Destacamos un estudio de Menard ${ }^{58}$, que tan sólo incluye 11 pacientes con tumores de próstata, que reciben radioterapia externa a diferentes dosis (66-76 Gy), con amifostina (1 gramo/20 ml) administrada endorectalmente 30 45 minutos antes de la radioterapia (los pacientes fueron instruidos a permanecer en decúbito prono, y retener la solución todo el intervalo, considerándose inadecuadas retenciones de menos de 30 minuto). En este estudio 6 pacientes $(55 \%)$ experimentaron toxicidad aguda grado 2 rectal $(5$ con aumento de la frecuencia rectal, que requirió loperamida, y 
1 con dolor hemorroidal que requirió corticoides tópicos). No se evalúa la toxicidad tardía. Los autores concluyen con que se necesitan más estudios y con mayor número de pacientes.

Conclusión: La amifostina es un radioprotector que reduce la severidad y duración de la xerostomía en pacientes que reciben radioterapia en tumores de cabeza y cuello, sin disminuir el control tumoral. A la vista de los estudios publicados parece que también puede disminuir la toxicidad intestinal en la irradiación pélvica, sin proteger el tumor. Sin embargo, debido a su elevado coste, y a que no está exenta de efectos secundarios, se necesitan más estudios clínicos, que definan mejor su papel, así como dosis y esquema óptimos, integración con las diferentes modalidades terapéuticas, y cómo mejorar su perfil de toxicidad. Por otra parte también es deseable que estos estudios incluyan valoraciones de calidad de vida, y por tanto de coste-efectividad.

\subsection{5-Aminosalicilatos (5-ASA)}

En sus diferentes formas: sulafasalazina, mesalamina, olsalazina, se utilizan como tratamiento anti-inflamatorio en la enfermedad inflamatoria intestinal (colitis ulcerosa y enfermedad de (rohn), y como antirreumático.

Poseen una acción inmunomoduladora, y son potentes inhibidores del Factor nuclear Kappa B (NF-KB). Así mismo poseen propiedades antioxidantes, eliminando los radicales libres, y reduciendo el daño oxidativo del DNA. Disminuyen el turn-over celular, y por último, parecen tener un efecto sobre la microflora intestinal.

En cuanto a su tolerancia, en el caso de la sulfasalacina, hasta el $45 \%$ de los pacientes presentan intolerancia, con dolor de cabeza, dispepsia, náuseas, vómitos, anorexia y fatiga, aunque se puede mejorar con la administración en la comida, reducción de dosis temporalmente, con una gradual incorporación posterior

Este perfil de toxicidad ha mejorado en los otros preparados, aunque hasta el $10 \%$ de los pacientes que presentan intolerancia a la sulfasalazina, la tienen también a los otros, incluyendo empeoramiento paradójico de la colitis en el caso de la mesalamina, o diarrea secretora en el caso de la olsalazina $^{59}$

Basado en la similitud de los procesos inflamatorios, mediados por citoquinas que se producen en la enfermedad inflamatoria intestinal y en la enteritis y proctitis radioinduci$\mathrm{da}^{60}$, se han utilizado estos compuestos en la prevención de las mismas, en algunos ensayos controlados, con resultado dispar:

En el estudio francés de Resbeut ${ }^{61}$, se randomizan 153 pacientes portadores de tumores de próstata y utero, y sometidos a irradiación pélvica (rango:45-52 Gy), seguido de sobreimpresión, con radioterapia externa o braquiterapia, a recibir mesalazina (4 gramos/día), o placebo. Los pacientes son seguidos durante 3 meses. No se objetiva ninguna diferencia en el porcentaje de pacientes que presentan diarrea (69\% frente a 66\%), ni en la duración de la misma. Sólo se objetivó mejoría en el dolor abdominal (34\% frente a $51 \%$ ).

Por el contrario Kilic lleva a cabo otro estudio randomizado, a doble ciego, con sulfasalazina ${ }^{62}$, en 87 pacientes sometidos igualmente a irradiación pélvica (dosis de 46. 50 Gy en 23- 25 fracciones, a través de sólo dos campos AP-
PA). La dosis de sulfasalazina fue de 2 gramos/día durante todo el tratamiento radioterápico. Encuentra una mejoría de la diarrea (grado 1-4) durante el tratamiento de los pacientes que toman sulfasalazina (55\% frente a $86 \%$ ), de la del grado 2 fue del $27 \%$ al $49 \%$. Globalmente también mejoró la toxicidad grado 1-3, valorado de acuerdo con los criterios de la escala LENT-SOMA ( $80 \%$ frente a 93\%; $p=0.07$ ).

\subsection{Misoprostol}

La utilización del misoprostol (análogo de la prostanglandina PGE2), está basado en el efecto radioprotector que parecen tener las prostanglandinas, incrementando la supervivencia de las células clonogénicas (stem cells). Además, podría prevenir el vertido celular, y la liberación de la enzima lisosomal.

Otra teórica ventaja es la ausencia de toxicidad a las dosis que confieren máxima radioprotección. La utilización más habitual del misoprostol, es por su efecto protector gastrointestinal, en la prevención de las úlceras gástricas y duodenales radioinducidas, así como mucositis radioinducidas. En pacientes con tumores de próstata, se ha utilizado en forma de supositorios, a dosis de 400 microgramos, administrados 1 hora antes de la radioterapia, obteniéndose una reducción eficaz de la proctitis, aunque tanto la dosis, como el intervalo óptimo de administración se deben precisar en futuros ensayos clínicos ${ }^{63}$.

\section{Tratamiento de soporte}

\subsection{Piso abdominal superior}

La irradiación del piso abdominal superior se produce como consecuencia de la administración de radioterapia para el tratamiento de tumores del tracto digestivo superior lestómago, páncreas, vía biliar, hepatocarcinomas...).

Los órganos críticos abdominales que se ven afectados son fundamentalmente el estómago, el hígado, el intestino delgado y el páncreas. La prevención de la toxicidad es la clave, realizando una correcta planificación del tratamiento radioterápico, y una evaluación pormenorizada de los histogramas dosis/volumen cuando se realice radioterapia tridimensional conformada, para no superar dosis de tolerancia.

A través de la experiencia obtenida de la irradiación postoperatoria del cáncer gástrico, la publicación consenso al respecto, realiza las siguientes recomendaciones ${ }^{64}$.

1. Recomendaciones dietéticas, nutricionales, e hídricas con una estrecha evaluación ponderal que deberá ser al menos semanal. Se recomienda una dieta con escasos residuos, pobre en grasas y lactosa, a la que puede añadirse complementos nutricionales especiales (por ejemplo glutamina, arginina, alimentos probióticos y prebióticos). En los casos más graves se recomienda la administración de suplementos nutricionales parcialmente digeridos, poliméricos, hipercalóricos y enriquecidos con ácidos grasos de cadena corta (EPA y DHA)

2. Puede ser necesario la administración de antiémeticos antagonistas de los receptores de serotonina 5-HT3 o metoclopramida.

3. Tratamiento profiláctico de la hemorragia digestiva alta con bloqueadores de los receptores $\mathrm{H}-2, \mathrm{o}$ inhibidores de la 


\section{A. Pérez Escutia y cols.}

bomba de protones, que se debe mantener durante 1-2 años tras finalizar el tratamiento.

4. Debido al síndrome de malabsorción que se produce, se necesita suplementos de vitamina B12, hierro y calcio.

\subsection{Enteritis aguda}

Se produce en más del $70 \%$ de los pacientes sometidos a irradiación. Además de una dieta estricta pobre en residuos, se aconseja el uso de agentes antidiarreicos (como la loperamida) que pueden mejorar la diarrea, aunque no deben utilizarse cuando se sospeche obstrucción intestinal. Actúan produciendo una reducción de la frecuencia de los movimientos intestinales, enlenteciendo el tránsito intestinal, y mejorando la absorción de los ácidos biliares.

En ocasiones puede ser necesario además, el uso de analgésicos o antiespasmódicos para controlar el dolor abdominal.

El octreótido, análogo de la somatostatina, es un agente antidiarreico seguro y eficaz, que tiene un excelente perfil de seguridad. Administrado subcutáneamente a dosis de 100 microgramos/3 veces día, controla la diarrea moderada- severa asociada a radioterapia, e incrementa así, la posibilidad de realizar tratamientos más continuos, evitando las interrupciones del tratamiento. Su eficacia es superior a la de los agentes convencionales antidiarreicos ${ }^{65}$, pudiendo representar una opción en el manejo de la diarrea asociada a radioterapia, grado 2 o superior.

\subsection{Proctitis aguda}

De forma similar se realizarán cuidados dietéticos, y tratamientos analgésico y antidiarreico. Además en la proctitis aguda puede ser útil la aplicación tópica de antiinflamatorios (como los derivados del ácido aminosalićlico), o corticoides.

\subsection{Enteritis crónica}

Su incidencia no está bien definida, pero se considera que aproximadamente entre el $1.2 \%$ y el $15 \%$ de los pacientes sometidos a radioterapia abdominal y pélvica, desarrollarán enteritis crónica, siendo dosis dependiente (generalmente con dosis superiores a $45 \mathrm{~Gy}$ ).

Su curso es insidioso y progresivo, comenzando generalmente a los seis meses de la irradiación. Conlleva un gran deterioro de la calidad de vida de los pacientes, y puede limitar su supervivencia (si no existe recidiva tumoral presenta una supervivencia a los 5 años en torno al 70\%).

El tratamiento debe ser tan conservador como sea posible, debe estar dirigido a aliviar la sintomatología (diarrea, dolor abdominal, nauseas, pérdida de peso, obstrucción, fístulas y perforación intestinal), y mejorar la calidad de vida del paciente, no existiendo tratamiento específico, ni pauta estándar. Incluye los siguientes aspectos:

Recomendaciones dietéticas: Ya comentadas previamente, pudiendo llegar a ser necesario, fórmulas enterales predigeridas y suplementadas con diferentes nutrientes /glutamina) y ácidos grasos omega-3.

Agentes antidiarreicos y analgésicos: Como la loperamida, los opiáceos débiles, los antiespasmódicos, para mejorar la diarrea y el dolor abdominal.
Antibióticos: Pueden reducir los síntomas en pacientes que desarrollen sobrecrecimiento bacteriano. El tratamiento específico es preferible al tratamiento empírico, y como no están exentos de efectos secundarios, que en ocasiones semejan los mismos síntomas que la sobreinfección bacteriana (molestias abdominales y diarrea), es importante establecer un diagnóstico.

A semejanza de la enfermedad inflamatoria intestinal, antibióticos como el metronidazol o la ciprofloxacina pueden ser útiles.

Corticoides: Se han utilizado como tratamiento primario de la enfermedad inflamatoria intestinal, y también en los episodios de reagudización de la enteritis y proctitis rádica, por su potente acción antiinflamatoria.

En su prescripción debemos valorar riesgos/beneficios, ya que poseen importantes efectos secundarios. Su utilización en cursos cortos (menos de 3 semanas), a dosis bajas (< $60 \mathrm{mg}$ ( día de prenisolona), presenta menor riesgo ${ }^{59}$.

Oxígeno hiperbárico: La elevación de la presión local de oxígeno, se utiliza por su efecto angiogénico, que incrementa en 8-9 veces la densidad vascular de los tejidos blandos, estimula la formación de colágeno en los márgenes de la herida, inhibe el crecimiento bacteriano y preserva el tejido marginal en buenas condiciones de perfusión. Todo ello permite una reepitelización de la mucosa, mediante la creación de una red capilar alrededor del tejido dañado. Se necesitan unas 20 - 30 sesiones.

El problema que presenta es que el equipo necesario es caro y de difícil disponibilidad, fuera de centros especializados.

Puede ser una opción terapéutica válida en casos de daño intestinal crónico, refractario a otros tratamientos.

Nutrición parenteral domiciliaria: Puede llegar a ser necesaria en el $5 \%$ de los pacientes con enteritis severa. Se utiliza cuando se produce un fallo intestinal como resultado de la radioterapia, en el que la cirugía no es factible, las principales indicaciones son la obstrucción intestinal persistente y el síndrome de asa corta, aunque también puede estar indicada en el caso de malabsorción, fístula, y dismotilidad intestinal.

Puede ser una medida transitoria mientras se restaura la nutrición oral, lo que suele suceder en el $35 \%$ de los pacientes, pero en otros muchos casos es necesario durante toda su supervivencia. La supervivencia a los 3 años es aproximadamente del $65 \%$, siendo la principal causa de mortalidad la recurrencia tumoral (71\% de los casos). La supervivencia y las complicaciones asociadas al mismo, son similares a los otros pacientes tratados igualmente con nutrición parenteral. Las infecciones del catéter son las complicaciones más frecuentes, aunque tienen baja mortalidad ${ }^{60}$.

Cirugía: A pesar de las medidas conservadoras de la enteritis radioinducida, la cirugía suele ser necesaria en el 30\% de los pacientes. Las indicaciones suelen ser por ileo persistente, fistulización intestinal, y adhesiones masivas. Durante la misma se debe valorar muy bien la existencia de recidiva tumoral.

El pronóstico es variable puesto que la enfermedad es progresiva: la mortalidad operatoria oscila en torno al 5\%, siendo más elevada si el procedimiento se lleva a cabo con carácter urgente, y si se lleva a cabo resecciones. La morbilidad operatoria oscila en torno al 30\%, ocupando un lugar destacado la dehiscencia de la anastomosis, aunque no pa- 
rece influir la naturaleza de la cirugía (urgente o programada), o el tipo (resección o conservadora).

Durante el seguimiento, el $40 \%$ de los pacientes requieren reoperación, debido a la recurrencia de los problemas, siendo esta tasa mayor en el grupo de la cirugía inicial conservadora (50\% frente a $34 \%$ ). Si no existe recidiva tumoral, la supervivencia es de $85 \%$ en el primer año, y $69 \%$ a los 5 años de la cirugía, estando influenciada por la naturaleza de la cirugía inicial (51\% si es conservadora, frente a $71 \%$ si es resección) $)^{67}$.

\subsection{Proctitis crónica}

Su incidencia varía entre un 5-10\%, presentando historia clínica difícil de precisar, que viene determinada por la presencia de dolor, descarga mucosa rectal, tenesmo, urgencia, sangrado rectal, estenosis y fístulas. Como la enteritis, posee un carácter insidioso y progresivo, comienza antes de los dos años tras la radioterapia, produciendo un gran deterioro de la calidad de vida. En la actualidad no hay un tratamiento que se considere estándar, aunque se recomienda sea lo más conservador posible y ajustado a los problemas que el paciente presente.

Así mismo se indica una dieta baja en residuos, y analgésicos para el control del dolor. Cuando existe sangrado, con frecuencia es necesario aporte de hierro e incluso transfusiones de concentrados de hematíes.

Pueden ser también de utilidad los tratamiento tópicos como los derivados del ácido aminosalicílico, sucralfato en forma de enemas, y los corticoides, siendo interesante los nuevos corticoides tópicos, como la budesonida, propionato de fluticasone, etc, ya que han demostrado que mantienen el efecto farmacológico antiinflamatorio, con mínimo impacto a nivel sistémico ${ }^{59}$.

Electrocoagulación con argón plasma: Resulta un método eficaz para mejorar la proctitis refractaria a otros tratamientos médicos. Los hallazgos endoscópicos mucosos consistentes en friabilidad difusa, telangiectasias, ulceración y edema, suelen mejorar con esta técnica. Generalmente se aplica de forma localizada en una zona de telangiectasia sangrante, más que en toda la zona friable. A menudo se necesitan varias sesiones. Posteriormente se produce la cicatrización y re-epitelización de la zona con tejido normal. Suele mejorar el sangrado, las necesidades de transfusión y de ingesta de hierro. Como complicaciones se describe dolor rectal, tenesmo y diarrea a largo plazo $(19 \%)^{68}$

Aplicaciones con formalina: Mediante esta técnica se esclerosa la neovasculatura frágil de forma puntual, reduciendo el sangrado. Puede ser un método eficaz, aunque el método de aplicación no está estandarizado, y presenta efectos secundarios como ulceración, estenosis, incontinencia y dolor. Como sucede con la aplicación del oxígeno hiperbárico, al no recogerse en los estudios datos de calidad de vida, no se puede establecer su beneficio terapéutico ${ }^{69}$.

Cirugía: Puede llegar a ser necesaria en aquellos pacientes que presentan sangrado severo, o pérdida de control del esfínter, en un porcentaje pequeño de casos, se debe realizar una colostomía. Las inyecciones de colágeno se han utilizado para el tratamiento de la incontinencia rectal, pero es un tratamiento que puede ser arriesgado, al ser una zona comprometida por la irradiación, y haber riesgo de dehiscencia $^{70}$

\section{Bibliografía}

1. P.A. Mitchell, S, Waggoner S, Rotmensch, J, Mundt, A.J. Cervical cancer in the elderly treated with radiotherapy. Gynecol. Oncol 1998; 71:291- 298

2. Herold D, Hanlon A, Hanks G. Diabetes Mellitus: A predictor for late radiation morbility. Int J. Radiat Oncol Biol. Phys 1999; 43 (3): $475-479$

3. Miller A, Materson J, Nelson $\mathrm{H}$, et al. The incidence and clinical consequences of treatment-related bowel injury. Int J. Radiat. Oncol. Biol. Phys. 1999; 43 (4): 817- 825.

4. Green S, Stock R, Greenstein A. Rectal cancer and inflammatory bowel disease: Natural history and implications for radiation therapy. Int J. Radiat. Oncol. Biol. Phys. 1999; 44 (4): 835- 840

5. Willet C, Ooi C, Zietman A, Menon V, et al. Acute and late toxicity of patients with inflammatory bowel disease undergoing irradiation for abdominal and pelvic neoplasmas. Int J. Radiat. Oncol. Biol. Phys. 2000; 46 (4): 995- 998.

6. Maduro J.H, Pras E, Willemse P, Vries E. Acute and log- term toxicity following radiotherapy alone or in combination with chemotherapy for locally advanced cervical cancer. Cancer Treatment Reviews 2003; 29 (6): 471- 488.

7. Gómez Candela C, Rodríguez Suarez L, Luengo Pérez L M, et al. Factores de riesgo nutricional en el paciente oncológico sometido a tratamientos antineoplásicos. En ed. Glosa. Intervención nutricional en el paciente oncológico adulto. Grupo de trabajo de la Sociedad Española de Nutrición Básica y Aplicada (SENBA). Novartis S.A. 2003: 45- 61 .

8. Cosset J.M, Henry-Amar M, Burgers J $M$, et al. Late radiation injuries of the gastrintestinal tract in the $\mathrm{H} 2$ and $\mathrm{H} 5$ EORTC Hodgkin's disease trials: Emphasis on the role of exploratory laparotomy and fractionation. Radiotherapy and Oncology 1998; 13: 61- 68.

9. Ooi B, Tiandra J, Gren M. Morbidities of adjuvant chemotherapy and radiotherapy for resectable rectal cancer. Dis Colon Rectum 1999; 42: 403- 418.

10. Stockolm Rectal Cancer Study Group. Preoperative radiation postpones resurrences in operable rectal carcinoma: a prospective randomised trial. Cancer 1990; 66: 49- 55.

11. Frykholm GJ, Glimelius B, Páhlman L. Preoperative or postoperative irradiation in adenocarcinoma of the rectum: final treatment results of a randomized trial and an evaluation of late secondary effects. Dis Colon Rectum 1993; 36: 564- 572.

12. Mameghan H, Fisher R, Mameghan J, et al. Bowel complications after radiotherapy for carcinoma of the prostate: the volume effect. Int J Radiat Oncol Biol Phys 1990; 18: 315 320.

13. Herbert SH, Curran WJ, Solin U, et al. Decreasing gastrointestinal morbidity with the use of small bowel contrast during treatment planing for pelvic radiation. Int J Radiat Oncol Biol Phys 1991; 20: 835- 842.

14. Minsky B. Pelvic radiation therapy in rectal cancer: Technical considerations. Seminars in Radiation Oncology 1993; 3 (1): 42- 47.

15. Gallagher MJ, Brereton HD, Rostock RA, et al. A prospective study of treatment techniques to minimize the volume of pelvic small bowel with reduction of acute and late effects associated with pelvic irradiation. Int J Radiat Oncol Biol Phys 1986; 12: 1565- 1573

16. Caspers RJL, Hop WCJ. Irradiation of true pelvis for bladder 


\section{A. Pérez Escutia y cols.}

and prostate carcinoma in supine, prone, or trendelenburg position. Int J Radiat Oncol Biol Phys 1983; 9: 589- 593.

17. Olofsen- van Acht $M$, Van den Berg S, Quint, et al. Reduction of irradiated small bowel volume and accurate patient positioning by use of a belly board device in pelvic radiotherapy of gynecological cancer patients. Radiot Oncol 2001; 59: 87-93

18. Devereux DF, Chandler JJ, Eisenstat T, et al. Efficacy of an absorbable mesh in keeping the small bowel out of the human pelvis following surgery. Dis Colon Rectum 1988; 31: 17- 21.

19. Zelefsky MJ, D'Amico A. External beam radiation therapy in the management of localized and locally advanced prostate cancer. Refresh course. ASTRO 2004.

20. Dearnaley D, Khoo V, Norman A, et al. Comparison of radiation side-effects of conformal and conventional radiotherapy in prostate cancer: a randomised trial. The Lancet 1999; 353 (9149): 267- 272.

21. Zelefsky MJ, Fuks Z, Hunt M, et al. High- dose intensity modulated radiation therapy for prostate cancer: early toxicity and biochemical outcome in 772 patients. Int J Radiat Oncol Biol Phys 2002; 53 (5): $1111-1116$.

22. Snyder KM, Stock RG, Hong SM, Chi Lo Y, Stine NN. Definig the risk of developing grade 2 proctitis following 125 l prostate brachytherapy using a rectal dose-volume histogram analysis. Int J Radiat Oncol Biol Phys 2001; 50 (2): 335- 341

23. Nag S, Erickson B, Thomadsen B, et al. The American Brachytherapy Society recommendations for high-dose-rate brachytherapy for carcinoma of the cervix. Int J Radiat Oncol Biol Phys 2000; 48 (1): 201-211.

24. Nag S, Chao C, Erickson B, et al. The American Brachytherapy Society recommendations for low-dose-rate brachytherapy for carcinoma of the cervix.. Int J Radiat Oncol Biol Phys 2002; 52 (1): 33- 48

25. Ciao LR, Myerson RJ, Tepper JE. Late effects of radiation therapy on the gastrointestinal tract. Int J Radiat Oncol Biol Phys 1995; 31: 1213- 1236.

26. Vázquez Martinez C. Nuevos nutrientes en nutrición enteral. Nutr Hosp. 2000; 15: 69-74.

27. Klimberg VS, Salloum RM, Kasper M, et al. Oral glutamine accelerates healing of the small intestine and improves outcome after whole abdominal radiation. Arch Surg 1990; 125: 1040- 1045.

28. Ersin S, Tuncyurek P, Esassolak M, et al. The prophylactic and therapeutic effects of glutamine and arginine enriched diets on radiation-induced enteritis in rats. Journal of Surgical Research 2000; 89: 121- 125.

29. Huang EY, Leung SW, Wang CJ, et al. Oral glutamine to alleviate radiation- induced oral mucositis: A pilot randomized trial. Int J Radiat Oncol Biol Phys 2000; 46:535- 539.

30. Richards EW, Long CL, Pinkston JA, et al: The role of oral glutamine supplementation in the prevention of radiation-induced enterocolitis in prostate cancer patients. FASEB J 1992; 6: 1680 (abstr).

31. Yoshida S, Matsui M, Shirouzu Y, et al. Effects of glutamine supplements and radiochemotherapy on systemic immune and gut barrier function in patients with advanced esophageal cancer. Ann Surg 1998; 227: 485- 491.

32. Kozelsky TF, Meyers GE, Sloan JA, et al. Phase III double-blind study of glutamine versus placebo for the prevention of acute diarrhea in patients receiving pelvic radiation therapy. J Clin Oncol 2003; 21: 1669- 1674.

33. Hall JC, Heel K, McCauley R: Glutamine. Br J Surg 1996; 83: 305- 312 .
34. Nakao M, Ogura Y, Satake $S$, et al. Usefulnessof soluble dietary fiber for the Treatment of diarrea during enteral nutrition in elderly patients. Nutrition 2002; 18: 35- 39.

35. Delia $P$, Sansotta $G$, Donato $V$, et al. Prevention of rafiation-induced diarrea with the use VSL-3, a new high-potency probiotic preparation. Am J Gastroenterol 2002; 97(8): 2150- 2152.

36. Heys SD, Walker LG, Smith I, Emerin O: Enteral nutrition supplementation with keys nutrients in patients with critical illness and cancer: a metanalysis of randomized controlled clinical trials. Ann Surg 1999; 229: 467- 477.

37. Epstein JB, Wong FL. The efficacy of sucralfate suspension in the prevention of oral mucositis due to radiation therapy. Int J Radiat Oncol Biol Phys 1994; 28: 693- 698.

38. Henrikson R, Franzén L, Littbrand B. Effects of sucralfate on acute and late bowel discomfort following radiotherapy of pelvic cancer. J Clin Oncol 1992; 10 (6): 969- 975.

39. Elsaid A, Elassal S. Sucralfate with pelvic radiotherapy: sense or nonsense?. Proceedings of the 40th annual ASTRO meeting 1998; Abstract 2112.

40. Valls $A$, Pestchen I, Prats $C$, et al. Ensayo clínico multicéntrico doble ciego comparativo de sucralfato y placebo en la prevención de la diarrea secundaria a irradiación pélvica. Medicina Clínica 1999; 113 (18): 681- 684.

41. Materson JA, Bollinger JW, Sloan JA, et al. Sucralfate in the prevention of treatment induced diarrhea in patients receiving pelvic radiation therapy: A North Central Cancer Treatment Group phase III double-blind placebo controlled trial. J Clin Oncol 2000; 18: 1239- 1245.

42. Stellamans $K$, Lievens $Y$, Lambin, et al. Does sucralfate reduce side effects of pelvic radiation? A double-blind randomized trial. Radiotherapy and Oncology 2002; 65(2): 105- 108.

43. Kneebone A, Mameghan $\mathrm{H}$, Bolin $\mathrm{T}$, et al. The effect of oral sucralfate on the acute proctitis associated with prostate radiotherapy: a double-blind, randomized trial. Int J Radiat Oncol Biol Phys 2001; 51(3): 628- 635 .

44. O'Brien PC, Franklin Cl, Dear K, et al. A phase III double-blind randomised study of rectal sucralfate suspension in the prevention of acute radiation proctitis. Radiotherapy and Oncology 1997; 45: 117- 123.

45. Taal BG, Olmos RAV, Boot H, Hoefnagel CA. Assesment of sucralfate coating by sequential scintigraphic imaging in radiation-induced esophageal lesions. Gastrointestinal Endoscopy 1995; 45 (2): 109- 114.

46. Kneebone A, Mameghan H, Bolin T, et al. Effect of oral sucralfate on late rectal injury associates with radiotherapy for prostate cancer: A double-blind, randomized trial. Int J Radiat Oncol Biol Phys 2004; 60 (4): 1088- 1097.

47. O’Brien PC, Franklin C.I., Poulsen M, Joseph D, Spry N, Denham J. Acute symptoms, not rectally administered sucralfate, predict for late radiation proctitis: longer term follow-up of a phase III trial- Trans-Taman Radiation Oncology Group. Int J Radiat Oncol Biol Phys 2002; 54 (2): 442- 449.

48. Valencia J, Escó R, Bascón N, et al. Análisis de los resultados del tratamiento con orgoteína asociado a radioterapia. Rev Oncol 1999; 1 (suppl. 6): 307-312.

49. Valencia J, Velilla C, Urpegui A, et al. The efficacy of orgoteína in the treatment of acute toxicity due to radiotherapy on head and neck tumors. Tumori 2002; 88: 385-389.

50. Benyahia B, Campana F, Perderau B, et al. Effects of superoxide dismutase topical treatment on human skin radiofibrosis: a pathological study. Breast 1996; 5: 75- 81. 
51. Esco R, Valencia J, Coronel P, Carceller JA, Gimeno M, Bascón $\mathrm{N}$. Efficacy of orgotein in prevention of late side effects of pelvic irradiation: a randomized study. Int J Radiat Oncol Biol Phys 2004; 60 (4):1211- 1219.

52. Britzel MD, Wasserman HD, Henke $M$, et al. Phase III randomized trial of amifostine as radioprotector in head and neck cancer. J Clinic Oncol 2000; 18: 3339- 3345.

53. Antonadou $D$, Coliarakis $N$, Sinodinou $M$, et al. Randomized phase III trial of radiation treatment + amifostine in patients witn advanced-stage lung cancer. Int J Radiat Oncol Biol Phys 2001; 51: 915- 922

54. Kligerman MM, Liu $T$, Liu $Y$, et al: Interim analysis of a randomized trial of radiation therapy of rectal cancer with/without WR-2721. Int J Radiat Oncol Biol Phys 1992; 22: 799-802.

55. Dunst J, Semlin S, Pigorsch S, et al. Intermittent use of amifostine during postoperative radiochemotherapy and acute toxicity in rectal cancer patients. Strahlenther Onkol 2000; 176: 416421.

56. Athanassiou $\mathrm{H}$, Antonadou $\mathrm{D}$, Coliarakis $\mathrm{N}$, et al. Protective effect of amifostine during fractionated radiotherapy in patients with pelvic carcinomas: Results of a randomized trial. Int J Radiat Oncol Biol Phys 2003; 56 (4): 1154-1160.

57. Koukourakis $M$, Kyrias $G$, Kakolyris $S$, et al. Subcutaneous administration of amifostine during fractionated radiotherapy: a randomized phase II study. J Clin Oncol 2000; 18: 22262233.

58. Menard C, Camphausen K, Muanza T, et al. Clinical trial of endorectal amifostine for radioprotection in patients with prostate cancer: rationale and early results. Seminars in Oncology 2003; supp 1830 (6): 1-8

59. Navarro F, Hanauer SB. Treatment of inflammatory bowel disease: safety and tolerability issues. Am J Gastroenterology 2003; 98 (12) supp 1: S18-S23

60. Indaram AVK, Visvalingam V, Locke M, Bank S. Mucosal cytokine production in radiation-induced proctosigmoiditis compared with inflammatory bowel disease. Am J Gastroenterology 2000; 95 (5): 1221-1225.

61. Resbeut M, Marteau P, Cowen, et al. A randomized double blind placebo controlled multicenter study of mesalazine for the prevention of acute radiation enteritis. Radiotherapy and Oncology 1997; 44: 59- 63.

62. Kilic D, Egehan I, Özenirler S, Dursun A. Double-blind, randomized, placebo-controlled study to evaluate the effectiveness of sulphasalazine in preventing acute gastrointestinal complications due to radiotherapy. Radiotherapy and Oncology 2000; 57: 125-129.

63. Khan AM, Birk JW, Anderson JC, et al. A prospective randomized placebo-controlled double-blinded pilot study of Misoprostol rectal suppositories in the prevention of acute and chronic radiation proctitis symptoms in prostate cancer patients. Am J Gastroenterology 2000; 95 (8): 1961-1966.

64. Smalley S, Gunderson L, Tepper J, et al. Gastric surgical adjuvant radiotherapy consensus report: rationale and treatment implementation. Int J Radiat Oncol Biol Phys 2002; 52(2): 283293.

65. Yavuz MN, Yavuz AA, Aydin F, Can G, Kavgaci H. The efficacy of octreotide in the therapy of acute radiation-iduced diarrhea: a randomized controlled study. Int J Radiat Oncol Biol Phys 2002; 54(1): 195- 202.

66. Scolapio J, Ukleja A, Burnes J, Kelly D. Outcome of patients with radiation enteritis treated with home parenteral nutrition. Am J Gastroenterology 2002; 97(3): 662-666.

67. Regimbeau J, Panis Y, Gouzi J, Fagniez P and the French University Association for Surgical Research. Operative and long term results after surgery for chronic radiation enteritis. Am J Surgery 2001;182: 237- 242.

68. Villavicencio RT, Rex DK, Rahmani E. Efficacy and complications of argon plama coagulation for hematochezia related to radiation proctopathy. Gastrointest Endosc 2002, 55 (1): 704.

69. Denton A, Forbes A, Andreyev J, Maher EJ. Non surgical interventions for late radiation proctitis in patients who have received radical radiotherapy to the pelvis (Cochrane Review). In: The Cochrane Library, Issue 4, 2004. Chichester, UK: John Wiley\& Sons, Ltd.

70. Lehur PA, Glemain P, Bruley D, et al. Outcome of patients with an implanted artificial anal sphinter for severe faecal incontinence. A single institution report. Int J Colorectal Dis 1998; 13: 88. 